\title{
MAGNETIC FIELD TOPOLOGY IN SOLAR ACTIVE REGIONS
}

\author{
N. SEEHAFER \\ Arbeitsgruppe "Nichtlineare Dynamik" der Max-Planck-Gesellschaft an \\ der Universität Potsdam, Am Neuen Palais, Haus 19, O-1571 Potsdam, \\ Germany
}

\begin{abstract}
In solar active regions, over extended periods of time the plasma-magnetic field configuration evolves quasistatically through a sequence of nearly force-free equilibrium states. This evolution may be understood as the continual distortion of an existing equilibrium by wavelike disturbances propagating upward from the photosphere and subsequent fast relaxation to a new, neighbouring equilibrium. In the present paper the build-up of magnetic energy, which is presumably necessary for flares and other explosive events, during a quasistatic evolution is considered. If during the slow evolution the magnetic energy is increased, then the relaxation processes represent inverse cascades of energy. We study the conditions under which such cascades are possible within the framework of mean-field MHD. In contrast to the convection zone, where the dynamo for the global magnetic field of the Sun works, the solar atmosphere is convectively stable and the first order smoothing approximation justified. It turns out then that current helicity $(\mathbf{B} \cdot \nabla \times \mathbf{B})$ is an important quantity decisive for whether magnetic energy can be built up.
\end{abstract}

\section{INTRODUCTION}

In the theories of the solar magnetism a particular topological property of the magnetic and velocity fields, namely helicity, which is thought to arise as a consequence of the rotation of the Sun, is of fundamental importance. With $\mathbf{v}$, $\mathbf{B}, \mathbf{A}$ and $\mathbf{j}$ denoting fluid velocity, magnetic induction, magnetic vector potential and electric current density, the densities per unit volume of kinetic, magnetic and current helicity are defined by

$$
H_{K}=\mathbf{v} \cdot \nabla \times \mathbf{v} ; \quad H_{M}=\mathbf{A} \cdot \mathbf{B} ; \quad H_{C}=\mathbf{B} \cdot \nabla \times \mathbf{B} .
$$

It is generally accepted that rotation is the cause for the global magnetic field of the Sun. The solar rotation also may be the ultimate cause for the generation of dc currents in the atmosphere. Such currents are presumably the energy source for flares and also may play a role for the non-flare heating of the atmosphere.

In this paper the particular role of current helicity is considered. It proves to be an important quantity for the build-up of currents in the solar atmosphere. 


\section{QUASISTATIC EVOLUTION OF THE MAGNETIC FIELD}

The photosphere can to some extent be considered as a rigid wall for the superphotospheric layers of the atmosphere, since the Alfvén velocity of the medium above the photosphere is much higher than that of the deeper layers. Since, in addition, the magnetic energy dominates over all other energies, the plasma-magnetic field configuration above the photosphere evolves, except for times of explosive events, slowly through a sequence of force-free equilibrium states, characterized by the equation

$$
\nabla \times \mathbf{B}=\alpha_{f f} \mathbf{B},
$$

where $\alpha_{f f}$ denotes a pseudo-scalar which in general depends on position. The current helicity and $\alpha_{f f}$ are related by the equation

$$
H_{C}=\alpha_{f f} \mathbf{B}^{2} .
$$

The slow evolution may be understood as a continual distortion of the equilibrium by disturbances propagating upward from the photosphere and subsequent fast relaxation to a new, neighbouring equilibrium. The relaxation leads to a state of minimum energy under given photospheric boundary conditions and further constraints following from the nature of the relaxation process. Without resistivity, i.e., if the magnetic field is frozen into the plasma, the relevant variational problem is

$$
\begin{gathered}
\int_{V}(\nabla \times \mathbf{A})^{2} d V=\text { minimum, } \\
\delta \mathbf{A} \times \mathbf{n}=0 \text { on } \partial V, \\
\delta \mathbf{A}=\delta \xi \times(\nabla \times \mathbf{A}) \text { for some } \delta \xi \text { in } V,
\end{gathered}
$$

where $\mathbf{n}$ is the normal on the surface $\partial V$ of the considered volume $V$ and $\delta \xi$ the displacement of a fluid element. Equation (6) expresses the frozen-infield condition, Equation (5) fixes the distribution of the photospheric flux, and Equations (5) and (6) together fix the photospheric end points of magnetic field lines (the "field line connectivity"). This problem leads to the necessary condition

$$
\mathbf{j} \times \mathbf{B}=\mathbf{0} .
$$

The magnetic field relaxes to a general force-free state, in which the factor $\alpha_{f f}$ in general is not spatially constant and the relaxation takes place on the short Alfvén time-scale.

If there is a small amount of resistivity, the system may relax further, namely by Taylor relaxation (Taylor 1974). The corresponding variational problem is given by Equations (4) and (5) and the condition that the total magnetic helicity is conserved,

$$
\int_{V} \mathbf{A} \cdot(\nabla \times \mathbf{A}) d V=\text { const. }
$$


The final state is force-free with spatially constant $\alpha_{f f}$. Taylor relaxation, involving magnetic reconnection, takes place on a reconnection time-scale, which is intermediate between the Alfvén and diffusive time-scales.

\section{RELAXATION AS INVERSE CASCADE}

If during the slow evolution the magnetic energy is increased, then, obviously, the relaxation processes represent inverse cascades of energy. Such cascades can be described within the framework of mean-field MHD, in which it is usual to decompose all quantities into mean and fluctuating parts. Accordingly the mean value of the current helicity can be represented as the sum of the two contributions resulting from the mean and fluctuating magnetic fields, respectively,

$$
\left\langle\mathbf{B} \cdot \nabla \times \mathbf{B}>=\left\langle\mathbf{B}>\cdot \nabla \times<\mathbf{B}>+<\mathbf{B}^{\prime} \cdot \nabla \times \mathbf{B}^{\prime}>\right.\text {. }\right.
$$

Angular brackets always indicate averages and a prime fluctuations.

Magnetic and velocity fluctuations lead to a mean electromotive force

$$
\mathcal{E}=\left\langle\mathrm{v}^{\prime} \times \mathrm{B}^{\prime}\right\rangle
$$

which for a wide range of assumptions can be written in the form

$$
\mathcal{E}=\alpha<\mathrm{B}>-\beta \nabla \times<\mathrm{B}>
$$

with coefficients $\alpha$ and $\beta$ which are in general tensors. The first term describes the $\alpha$-effect, the second a turbulent diffusivity.

The coefficients $\alpha$ and $\beta$ are usually evaluated by applying the first order smoothing approximation (FOSA) (cf. Moffatt 1978, Krause and Rädler 1980). This approximation is justified if

(a) $R m \ll 1$ or

(b) $\left|\mathbf{v}^{\prime}\right| \ll \lambda / \tau=S$ or

(c) $\left|\mathbf{B}^{\prime}\right| \ll \mid<\mathbf{B}>1$.

Here $R m$ is the magnetic Reynolds number, $\lambda$ and $\tau$ are length- and time-scales typical of the fluctuations, and $S$ is the Strouhal number. Clearly, condition (a) is not satisfied in the solar plasma. With respect to the Strouhal number one has to distinguish between conventional turbulence and wave turbulence: $S \approx 1$ for conventional turbulence and $S \ll 1$ for wave turbulence. In the latter case a disturbance does not lead to the onset of convection but only to a wave. So $S \approx 1$ in the convectively unstable convection zone and $S \ll 1$ in the convectively stable atmosphere. Condition (c) finally seems to be satisfied in individual active regions, whereas on the global scale (and the time-scale of the activity cycle) the mean field is possibly even small compared to the fluctuations. So FOSA seems to be much more justified for the atmosphere than for the convection zone, to which it usually is applied in the context of dynamo theory.

Now let FOSA be valid in the atmosphere and let the photosphere be a perfectly conducting rigid wall with respect to the mean field (the mean-field Poynting flux is assumed to vanish), so that electromagnetic energy can penetrate 
the photosphere only on the scale of the fluctuations. Then (Rädler and Seehafer 1990, Seehafer 1992)) under the additional assumptions that

(i) $\langle\mathbf{v}>=0$,

(ii) $\mathbf{v}^{\prime}$ describes a homogeneous, steady and isotropic turbulence,

the energy content of $\langle\mathbf{B}\rangle$ in the volume above the photosphere can grow (or at least be stationary) only if there is some (sufficiently large) subvolume in which

(1) $<\mathbf{B}^{\prime} \cdot \nabla \times \mathbf{B}^{\prime}>\neq 0$ and $<\mathbf{B}>\cdot \nabla \times<\mathbf{B}>\neq 0$,

(2) $<\mathbf{B}^{\prime} \cdot \nabla \times \mathbf{B}^{\prime}>$ and $\langle\mathbf{B}>\cdot \nabla \times<\mathbf{B}>$ have opposite signs,

(3) $\left|<\mathbf{B}^{\prime} \cdot \nabla \times \mathbf{B}^{\prime}\right\rangle|>|<\mathbf{B}>\cdot \nabla \times<\mathbf{B}>\mid$.

It seems interesting to note that for the inverse cascade not only the small-scale but also the large-scale field must possess current helicity (of appropriate sign), in order to be able to pick up the energy from the small scale.

\section{DISCUSSION}

According to a recent study (Seehafer 1990) comparing force-free magnetic fields calculated from photospheric magnetograms with chromospheric and coronal observations, mainly in $\mathrm{H} \alpha$ and EUV lines, $H_{C}$ is predominantly negative in the northern and positive in the southern hemisphere. This is just what would be expected from the theoretical results presented in this paper. So e.g., in order that a significant amount of magnetic energy can be built up within an active region, the current helicity of the fluctuations must have a predominant sign over a sufficiently long period of time. If this would happen only by chance, the energy build-up should not be so typical of active regions as it obviously is. A systematic generation of helicity as a consequence of the solar rotation, namely by the action of Coriolis forces, seems more likely. Then, however, the current helicity should have the same sign in all regions of the same hemisphere and opposite signs in northern and southern hemisphere.

So it seems most interesting to analyse the available longitudinal and vector magnetograms in order to confirm or disprove the above sign rule.

\section{REFERENCES}

Krause, F. and Rädler, K.-H. 1980, Mean-Field Magnetohydrodynamics and Dynamo Theory, Akademie-Verlag.

Moffatt, H.K. 1978, Magnetic Field Generation in Electrically Conducting Fluids, Cambridge Univ. Press.

Rädler, K.-H. and Seehafer, N. 1990, in Topological Fluid Mechanics, ed. H.K. Moffatt and A. Tsinober, Cambridge Univ. Press, p. 157.

Seehafer, N. 1990, Solar Phys., 125, 219.

Seehafer, N. 1992, Solar Phys., submitted.

Taylor, J.B. 1974, Phys. Rev. Letters, 33, 1139. 